
allemande

50-1| 2018

Regards franco-allemands sur la justice dans la construction européenne | Les élections législatives de 2017 en Allemagne et en Autriche

\title{
Envoyer des jeunes Algériens en France, en RFA et en Suisse
}

Trois reflets de la guerre d'indépendance algérienne en Europe

Lucas Hardt

\section{(2) OpenEdition}

Édition électronique

URL : https://journals.openedition.org/allemagne/664

DOI : 10.4000/allemagne.664

ISSN : 2605-7913

Éditeur

Société d'études allemandes

\section{Édition imprimée}

Date de publication : 30 juin 2018

Pagination : 185-197

ISSN : 0035-0974

\section{Référence électronique}

Lucas Hardt, "Envoyer des jeunes Algériens en France, en RFA et en Suisse », Revue d'Allemagne et des pays de langue allemande [En ligne], 50-1 | 2018, mis en ligne le 30 juin 2019, consulté le 04 juin 2022. URL : http://journals.openedition.org/allemagne/664; DOI : https://doi.org/10.4000/allemagne. 664 


\section{Envoyer des jeunes Algériens en France, en RFA et en Suisse Trois reflets de la guerre d'indépendance algérienne en Europe}

- Lucas Hardt *

\section{Introduction}

Suite au début de la guerre d'indépendance algérienne, une vaste propagande pour le maintien de l'Algérie française était mise en œuvre. Cette propagande, dont les auteurs agissaient soit pour leur propre compte, soit dans le cadre d'une fonction de l'État français, avait pour vocation de lier comme un seul ensemble la population française et algérienne des deux côtés de la Méditerranée ${ }^{(1)}$. Les jeunes et adolescents n'échappèrent pas à cette volonté et on observera au contraire comment ils se retrouvèrent à la fois objets et acteurs malgré eux de cette stratégie de propagande ${ }^{(2)}$. En se fondant sur l'exemple des séjours organisés pour des enfants algériens en métropole, en République fédérale d'Allemagne et en Suisse durant la guerre d'Algérie, cet article montrera que la portée de l'action psychologique pro Algérie française ne se limitait ni aux frontières de l'Algérie ni à celles de la métropole.

Pour ce qui est de la Suisse, on sait que les activités des rebelles algériens sur son territoire ainsi que la présence d'une importante communauté française ont conduit

* Docteur en histoire et chercheur associé au Centre Marc Bloch.

L'auteur tient à remercier Camille Colleu, Julien Ségol et Hélène Lenoir pour leurs corrections de la version française du texte.

1 Au mois d'octobre 1955, une circulaire du ministre de l'Intérieur incitait les préfets de la métropole à une action répressive, humaine et psychologique dans leurs départements contre la propagande communiste visant à discréditer la présence française en Afrique du Nord. Selon la volonté de Maurice Bourgés-Maunoury, cette action devait également s'appliquer dans les écoles. Le Ministre de l'Intérieur à Messieurs les Inspecteurs Généraux de l'Administration en mission extraordinaire, Messieurs les préfets, le 8 octobre 1955, p. 4, Archives départementales de la Moselle (AdM) 252 W 16.

2 Ce sujet a déjà été traité dans un autre article de l'auteur publié en allemand et n'abordant que la dimension franco-algérienne des jumelages et des transferts d'enfants algériens en métropole durant la guerre d'Algérie. Lucas HARDT, "Ferienlager gegen die Rebellion. Jumelages und Colonies de vacances im Algerienkrieg (1959-1962) », Journal of Modern European History, 11 (2013), p. 351-373. 
à ce que l'on accorde aux affrontements sur la question algérienne une attention toute particulière. Ce fut notamment le cas au moment de la célèbre «affaire Dubois » qui, en 1957, révélait au grand public d'importantes activités d'espionnage des services secrets français en Suisse ${ }^{(3)}$. Par la suite, l'intérêt helvétique prêté à ce conflit, que le FLN sut progressivement internationaliser, releva principalement de la politique étrangère. $\mathrm{Ce}$ fut notamment après le discours du général de Gaulle sur l'autodétermination algérienne, en septembre 1959, que les voyages de journalistes suisses en Algérie se multiplièrent et que le sujet se trouva de plus en plus présent dans les médias du pays ${ }^{(4)}$. C’est dans ce contexte d'une attitude qui dans son ensemble était devenue plutôt favorable à la politique française en Algérie que l’Union des Français en Suisse allait se lancer dans l'organisation de séjours d'enfants algériens en Suisse.

La position de la République fédérale d'Allemagne par rapport à la question algérienne s'avérait particulièrement délicate ${ }^{(5)}$. Sur le plan de sa politique étrangère, le gouvernement de Bonn privilégiait le maintien et le développement de bonnes relations avec Paris sans pour autant pouvoir ignorer le soutien international grandissant dont jouissait le $\mathrm{FLN}^{(6)}$. Au niveau de sa politique intérieure, le gouvernement Adenauer fut confronté à l'entrée, presque toujours clandestine, de plusieurs milliers d'Algériens en RFA fuyant surtout le lourd climat de répression anti-algérienne en France. La question de leur droit d'asile en tant que réfugiés politiques potentiels faisait l'objet de nombreuses discussions, surtout parce que les services secrets français n'avaient de cesse de demander une surveillance aiguë de ceux-ci, voire de réclamer l'extradition de certains. Dans cette posture délicate, les représentants de l'État ouest-allemand assumaient pour la plupart de répondre aux attentes de l'allié français en essayant toutefois d'éviter de prendre publiquement position sur la question algérienne.

La comparaison de ces trois cas illustrera d'importantes différences en ce qui concerne les motivations qui ont présidé à l'organisation de ces voyages ainsi que les conditions de séjour des enfants. Ces disparités révèlent que les acteurs impliqués dans cette guerre coloniale s'appropriaient les différents clivages du conflit selon leurs propres interprétations et stratégies.

\section{Gagner les cœurs pour l'Algérie française. Des jeunes Blidéens en Moselle}

En métropole, les séjours de jeunes Algériens durant la guerre d’Algérie étaient organisés surtout par trois canaux. En premier lieu, des organisations de différentes orientations politiques et religieuses prenaient en charge des enfants d'immigrés algériens qui vivaient en métropole et pour la plupart dans des conditions particulièrement précaires, afin qu'ils fassent un séjour dans une colonie de vacances à

3 Damien Carron, La Suisse et la guerre d'indépendance algérienne (1954-1962), Lausanne, Éditions Antipodes (coll. Histoire), 2013, p. 152-197.

4 Ibid., p. 252-254.

5 Jean-Paul Cahn, Klaus-Jürgen Müller, La République fédérale d'Allemagne et la guerre d'Algérie (1954-1962). Perception, implication et retombées diplomatiques, Paris, Le Félin (coll. histoire et sociétés), 2003.

6 Mathilde von Bülow, West Germany, Cold War Europe and the Algerian War, Cambridge University Press (coll. New studies in European history), 2016. 
la campagne ${ }^{(7)}$. Par ailleurs, en métropole comme en Algérie, des syndicats et des entreprises offraient la possibilité à leurs travailleurs, indépendamment de leur origine, d'envoyer leurs enfants dans des colonies de vacances durant l'été. Finalement, des séjours de jeunes Algériens dans des colonies de vacances en métropole étaient organisés dans le cadre des jumelages et parrainages ${ }^{(8)}$, d'abord entre communes puis entre régions françaises et algériennes ${ }^{(9)}$. Un des premiers partenariats communaux franco-algériens fut le parrainage entre Metz et Blida, conclu en 1956. Ce parrainage donna lieu à un premier voyage de 13 jeunes Blidéens à Metz au cours de l'été de la même année. Soucieux de la contribution de ce projet à la bonne entente entre Européens ${ }^{(10)}$ et Algériens, les conseillers municipaux messins se montrèrent déçus d'apprendre que ce groupe était exclusivement composé de jeunes Européens. Selon le maire de Metz, Raymond Mondon, qui avait tenu à accueillir personnellement les jeunes participants, les cinq jeunes Algériens annoncés auraient été décommandés "pour des raisons de pression politique, morale ou autres» ${ }^{(11)}$.

Malgré ce premier échec, le maire de Metz demeura déterminé à poursuivre les activités dans le cadre de ce jumelage. Par ses divers voyages à Blida au cours des années 1956 et 1957, Raymond Mondon affirmait sa volonté de consolider le parrainage de la ville de $\mathrm{Metz}^{(12)}$. Au début de l'année 1958, une campagne de solidarité était lancée dans le chef-lieu de la Moselle, grâce à laquelle on allait collecter plus d'une tonne de marchandises et 1,5 million de francs. Le député-maire se chargea personnellement de remettre l'ensemble de ces dons à son homologue blidéen, Charles Baujard ${ }^{(13)}$. Accompagné d'une délégation de 18 personnes ainsi que de son épouse, Raymond Mondon devait profiter de ce voyage pour visiter un «secteur opérationnel» de l'armée française et rencontrer des anciens combattants algériens de la Deuxième Guerre mondiale, ainsi que les écoliers d'un village des environs de Blida ${ }^{(14)}$.

7 Pour la plupart, cet engagement se réalisait dans le cadre d'initiatives privées ou semi-privées œuvrant pour une action «sociale et humanitaire» vis-à-vis des «Nord-africains» vivant en métropole et s'inspirant souvent de la «mission civilisatrice». Voir Elizabeth SLOAN, Algerian Youth in the colonies de vacances during the Algerian War, https://www.google.de/search?q=Elizabeth+Sloan+Algerian+ Youth\&ie=utf-8\&oe=utf-8\&client=firefox-b\&gfe_rd=cr\&ei=gVDvWNLGNIHVXu28qOAI (13 avril 2017). Pour une analyse de l'action sociale pour les Algériens en métropole durant la guerre d'Algérie, voir Amelia H. Lyons, «The Civilizing Mission in the Metropole. Algerian Immigrants and the Politics of Adaptation during Decolonization", Geschichte und Gesellschaft, 32 (2006), p. 489-516.

8 Les relations franco-algériennes de la première heure furent pour la plupart appelées "parrainages " ce qui faisait apparaître la hiérarchie coloniale au sein de ses partenariats. Suite à l'initiative du premier ministre Michel Debré dans ce domaine en 1959 et à la politique de la «fraternisation» francoalgérienne annoncée par le général de Gaulle depuis 1958, les relations fonctionnaient pour la plupart sous l'appellation de «jumelages».

9 Voir à ce sujet, Lucas HARDT, «Ferienlager gegen die Rebellion» (note 2).

10 Par Européens nous comprenons des personnes qui à l'époque étaient désignés en tant que «Français de souche européenne».

11 Procès-verbal de la séance du 26 octobre 1956 du conseil municipal de Metz, Archives municipales de Metz, dMSC 171 - 1956.

12 Jean Verchin, «Les maires de France à Blida», Revue trimestrielle de Blida, 3/15 (1957), p. 9-10, Archives municipales de Metz, 5 S 47.

13 Le Républicain Lorrain, 8 mars 1958.

14 Le Républicain Lorrain, 6 mars 1958. 
Dans cette première phase des jumelages et parrainages franco-algériens entre 1956 et 1959, certains contemporains pouvaient avoir le sentiment d'assister en fait à un échange franco-français. Jusqu'en 1959, aucune des anciennes communes mixtes d'Algérie $^{(15)}$ n'avait fait l'objet d'un parrainage franco-algérien ${ }^{(16)}$. Cependant, la nouvelle importance donnée à la «fraternisation» franco-algérienne par le gouvernement de Michel Debré se fit également ressentir au niveau de la ville mosellane. Suite à la proposition du Premier ministre, le conseil général du département acceptait de se jumeler à l'arrondissement de Blida, créant ainsi une nouvelle structure d'échange qui allait éclipser celle du parrainage. C'est ainsi qu'en 1960, un groupe de 98 enfants blidéens, dont $90 \%$ étaient présentés comme musulmans, allait passer un mois dans des colonies de vacances du département. Durant le mois de juin, les 50 garçons et 48 filles étaient répartis dans dix colonies de vacances différentes. Celles-ci avaient reçu de la part du conseiller technique pour les affaires musulmanes (CTAM) de la Moselle des recommandations concernant d'éventuels besoins ou réquisitions spécifiques pour l'accueil des enfants algériens et leur séjour en Lorraine ${ }^{(17)}$.

L'été suivant, la Moselle devait de nouveau accueillir un groupe de 99 enfants venus de Blida pour un séjour d'un mois en Moselle. Répartis sur dix colonies de vacances, ils furent suivis de près par le CTAM, François Guigue, pendant toute la durée de leur séjour en métropole ${ }^{(18)}$. Cette implication des CTAM, agissant comme les «fers de lance du paternalisme colonial ${ }^{(19)}$, révèle combien l'enjeu central de ces colonies de vacances franco-algériennes résidait en fait dans une volonté d'enthousiasmer les jeunes Algériens pour une vie dans la métropole présentée à travers le prisme de sa modernité et de sa capacité à respecter leurs "particularités culturelles». En ce sens, c'est avant tout le contact avec des enfants d'origine européenne du même âge qui devait jouer un rôle majeur ${ }^{(20)}$.

Avec l'empire colonial allait s'effondrer progressivement aussi le réseau des jumelages et parrainages franco-algériens. Aujourd'hui à Metz, hormis le nom d'une rue et

15 Ces communes avec une majorité d'habitants algériens avaient un statut juridique spécifique afin d'assurer la domination politique de la minorité des habitants non-musulmans. Voir Raphaëlle BRANCHE, L'embuscade de Palestro. Algérie 1956, Paris, Armand Colin (coll. Le Fait guerrier), 2010, p. 233.

16 Étude sur les parrainages métropole-Algérie effectuée par l’État-major, $10^{\mathrm{e}}$ région militaire $-5^{\mathrm{e}}$ bureau à la suite de la tournée d'information du «centre des jeunes patrons» (s.d. résumé: mai 1959), p. 2, Service historique de l'armée de Terre (SHAT), 1 H 2462.

17 Rapport trimestriel de François Guigue, conseiller technique pour les affaires musulmanes à la préfecture de la Moselle du $1^{\text {er }}$ avril au 30 juin 1960, p. 25, AdM 297 W 66.

18 L’Administrateur des Services civils d'Algérie, conseiller technique pour les affaires musulmanes à M. le Préfet du Service des affaires musulmanes le 28 septembre 1961, Archives du ministère des Affaires étrangères (AMAE), Secrétariat d'État aux affaires algériennes 1959-1967, carton 85.

19 Françoise de BARros, "Contours d'un réseau administratif “algérien” et construction d'une compétence en "affaires musulmanes". Les conseillers techniques pour les affaires musulmanes en métropole (1952-1965) », Politix, 4 (2006), n 76, p. 97-117.

20 Dans son rapport concernant l'été 1961, le CTAM en Moselle souligne et se félicite que le groupe de jeunes Algériens déjà divisé en groupes d'environ dix enfants répartis sur les différentes colonies du département ait été ensuite encore redivisé de façon à ce que les jeunes de ces groupes soient répartis dans des camps de vacances différents et se mêlent ainsi au plus grand nombre possible d'enfants métropolitains. L’Administrateur des Services civils d'Algérie, conseiller technique pour les affaires musulmanes, à M. le Préfet du Service des affaires musulmanes (note 18). 
celui d'un centre d'innovation, rien ne rappelle le passé spécifique qui liait cette ville à Blida. Tout comme l'impact de la guerre d'Algérie en Moselle, le parrainage MetzBlida semble avoir été effacé de la mémoire collective.

\section{Action humanitaire ou psychologique? Voyages de mineurs algériens en Suisse 1959-1962}

Dans la foulée des activités conclues au sein des jumelages et parrainages francoalgériens, des expatriés français préparèrent des voyages de jeunes Algériens aussi en Suisse. En 1959, l’Union des Français en Suisse créait l'œuvre indépendante "Pour Aïn-Touta ${ }^{(21)}$ qui, par la suite, allait organiser des séjours individuels et des colonies de vacances pour des enfants algériens sur le territoire helvétique. Selon un de ses anciens membres, l'objectif aurait été de «s'associer au mouvement de jumelage des localités métropolitaines avec les nouveaux villages qu'on créait en Algérie, pour remplacer les camps de regroupements militaires " ${ }^{(22)}$. C'est ainsi que l'Union des Français en Suisse allait accepter de parrainer trois Centres de regroupement du département d'Orléansville en Algérie ${ }^{(23)}$ et que «Pour Aïn-Touta» se mit à recruter des Français vivant en Suisse pour accueillir au moins un enfant algérien pour un séjour dans leur famille ${ }^{(24)}$.

Ainsi, durant l'été 1960, les premiers enfants et adolescents algériens firent un séjour en Suisse pouvant ainsi quitter pour un temps les camps de l'armée française en Algérie ${ }^{(25)}$. À l'instar des voyages organisés dans le cadre des jumelages et parrainages franco-algériens, les autorités françaises prenaient en charge les frais de voyage des enfants jusqu'en Suisse ${ }^{(26)}$. En revanche, c'est l'organisation «Pour Aïn-Touta» qui prenait à sa charge tous les frais de voyage, de séjour et d'assurance des enfants sur le territoire suisse ${ }^{(27)}$.

Cette idée, consistant à faire venir des enfants nécessiteux d'Algérie en Suisse, avait auparavant déjà été discutée par certains médias helvétiques. Les conditions de vie des détenus Algériens dans les camps militaires français faisaient déjà l'objet de discussions et de reportages à la radio ainsi que dans certains journaux suisses. L'arrivée des premiers enfants algériens en Suisse avait été précédée de plusieurs appels à la

21 Aïn-Touta était une ancienne commune mixte dans l'Algérie coloniale.

22 Pierre Piqué, «La Fédération des Français en Suisse. Quelques notes d'histoire», Bulletin de la Société Française de Fribourg, n 46 (nº spécial), septembre 1994, p. 16, BNF, 4 JO 74847 - 1994. L'auteur de cet article avait participé à la guerre d'Algérie en tant que volontaire dans l'armée française de 1958 à 1960. Avant d'écrire cet article, il avait été, par la suite, président de la Société française de Fribourg en 1987.

23 Le Ministre d'État chargé des Affaires algériennes à Monsieur Jean-Claude Jaccard, Président de la «Terre des Hommes», s.d. (envoyé avant le 15 février 1961), AMAE, Secrétariat d'État aux affaires algériennes 1959-1967, carton 86.

24 Le Professeur Vicaire, Président de «Pour Aïn-Touta », à Monsieur le Ministre d'État chargé des Affaires algériennes, le 6 juin 1961, AMAE, Secrétariat d'État aux affaires algériennes 1959-1967, carton 85.

25 Le Ministre d'État chargé des Affaires algériennes à Monsieur Jean-Claude Jaccard (note 23).

26 Lettre sans auteur adressée au Révérend Père Vicaire Albertinum, Fribourg en Suisse, le 17 février 1962, AMAE, Secrétariat d'État aux affaires algériennes 1959-1967, carton 86.

27 Lettre de R.P. Vicaire, Président de «Pour Aïn-Touta», sans destinataire du $1^{\text {er }}$ février 1962, AMAE, Secrétariat d'État aux affaires algériennes 1959-1967, carton 86. 
solidarité ${ }^{(28)}$. Néanmoins, le nombre total de ces séjours semble avoir été assez limité. En 1961, «Pour Aïn-Touta» ne parvint à recruter que 22 personnes prêtes à recevoir au moins un enfant algérien chez elles ${ }^{(29)}$.

Cela n'empêcha pas une seconde organisation helvétique de s'engager dans la même action. À peine fondée par Edmond Kaiser et Jean-Claude Jaccard, l'association «Terre des Hommes", à l'époque aussi connue sous la dénomination de «Mouvement de secours immédiat et direct à l'enfance misérable du monde», établit un contact avec le Gouvernement général d'Algérie ${ }^{(30)}$. Au mois de septembre 1960, son président Jean-Claude Jaccard proposa aux autorités françaises d’accueillir des enfants algériens malades pour un séjour de convalescence en Suisse. Son idée était de faire venir chaque mois des convois de vingt enfants en Suisse et de les placer ensuite, selon leur état de santé, dans des familles, dans des maisons d'enfants, ou dans des hôpitaux. Cependant, la réaction du Gouvernement général d'Algérie fut dans un premier temps empreinte de scepticisme. En novembre 1960, son délégué général Vaujour notait:

«D’abord très touché par cette proposition humanitaire, j’ai pensé ensuite qu'il était sage de s'entourer de garanties pour éviter les inconvénients possibles sur le plan politique. 1. L'hébergement dans des familles suisses peu favorables à la France qui ne manqueraient pas de montrer en spectacle les enfants algériens déficients pour étayer une propagande nocive. 2. La promiscuité possible avec des enfants algériens appelés du Maroc ou de la Tunisie. 3. Le précédent créé pour des œuvres d'autres puissances étrangères moins favorables à notre égard, qui pourraient faire une offre semblable difficile à refuser après notre acceptation donnée à l'œuvre “Terre des Hommes” suisse» ${ }^{(31)}$.

Malgré les risques potentiels évoqués de cette action humanitaire, "Terre des Hommes» obtint temporairement en novembre 1960 l'autorisation de recevoir des enfants algériens en Suisse, à condition que ces mineurs restent sous le contrôle des moniteurs relevant des services publics algériens durant leur séjour ${ }^{(32)}$. Après s'être renseigné auprès de l'ambassade française en Suisse sur d'éventuelles activités politiques de «Terre des Hommes» et son président Jaccard ${ }^{(33)}$, le Délégué général d'Algérie finit par donner son accord définitif à ce sujet en février 1961. Comme dans les rapports avec «Pour Aïn-Touta» les autorités françaises s'engageaient à prendre en charge le voyage en Suisse des enfants et des deux assistantes sociales qui devaient les accompagner alors que «Terre des Hommes» assurait l'intégralité des frais du séjour

28 Les conditions de vie pour les détenus algériens dans les camps militaires français faisaient l'objet de discussions et de reportages à la radio et dans certains journaux suisses. Edmond KAISER, Émission du Schweitzer Radio und Fernsehen (SRF) du 11 avril 1991, http://www.srf.ch/player/tv/-/video/edmondkaiser?id=ff147c28-38e0-4d46-a798-55950a700030 (18.01.2015).

29 Le Professeur Vicaire, Président de «Pour Aïn-Touta», à Monsieur le Ministre d'État chargé des Affaires algériennes (note 24).

30 Le Ministre d'État chargé des Affaires algériennes à Monsieur Jean-Claude Jaccard (note 23).

31 Le Délégué général du gouvernement en Algérie, Cabinet civil, Vaujour au Secrétaire Général, Affaires algériennes, le 4 novembre 1960, AMAE, Secrétariat d'État aux affaires algériennes 1959-1967, carton 86.

32 Ibid.

33 Le Ministre des Affaires étrangères à Monsieur le Premier Ministre, Secrétariat général aux Affaires algériennes, le 21 novembre 1960, AMAE, Secrétariat d'État aux affaires algériennes 1959-1967, carton 86. 
sur le territoire Suisse ${ }^{(34)}$. C'est dans ce cadre qu'environ 300 enfants algériens, dont les plus jeunes étaient âgés de cinq ans, allaient quitter un Centre de regroupement d'Orléansville pour séjourner dans les environs de Lausanne ${ }^{(35)}$.

Du point de vue des autorités françaises et dans le contexte de la guerre d'Algérie, le fait d'envoyer des enfants algériens à l'étranger ne signifiait pas pour autant s'abstenir de les contrôler. Avant même que les premiers enfants soient accueillis à Lausanne, le ministère des Affaires étrangères avait mis en garde les autorités responsables quant à cette ville: «Lausanne étant le principal centre des activités arabes en Suisse, il faudrait éviter que certains groupements ne puissent profiter du séjour des enfants pour les endoctriner au profit du FLN ${ }^{\left({ }^{(6)}\right.}$. C'est de telles préoccupations d'ordre politique que découla la consigne stricte de veiller à ce que les enfants ne soient jamais dispersés et gardés à l'écart des grandes villes. Pour assurer le respect de ce règlement «deux assistantes sociales rompues au travail en milieu musulman» étaient d'une certaine manière chargées de remplir une partie des fonctions du CTAM en Moselle, à savoir d'accompagner le groupe afin d'assurer leur encadrement, la correspondance avec les familles, sans oublier de "résoudre les petits problèmes qui peuvent naître des différences de psychologie ${ }^{(37)}$.

Mise à part l'éventualité d'effets politiques négatifs sur les enfants durant leur séjour, les autorités françaises redoutaient également que la présence de ces témoins de la guerre en Algérie ne puisse influencer l'opinion publique suisse. C'est pour cette raison que le ministre des Affaires étrangères incita à «veiller à n'envoyer en Suisse que des enfants dont les récits ne soient pas susceptibles d'impressionner fâcheusement les personnes qui les accueilleront " ${ }^{(38)}$. C'est précisément dans ce sens que le ministre d'État chargé des Affaires algériennes formula au mois de février 1961 une vive critique à l'égard d'un convoi de 21 enfants partis de Khenchela pour la Suisse. À ces enfants arrivés à Lyon "dans une tenue misérable et sans aucun bagage», le CTAM de la préfecture du Rhône s'était vu dans l'obligation de fournir, par le biais de la Croix rouge, des vêtements avant leur transfert à Lausanne. Afin de veiller à la bonne image de la France à l'étranger, le ministre ordonna que l'on veille à ce que les enfants soient

34 Le Délégué général en Algérie à Monsieur le Ministre d'État chargé des Affaires algériennes, le 18 février 1961, AMAE, Secrétariat d'État aux affaires algériennes 1959-1967, carton 86.

35 En janvier 1962, on comptait déjà 254 enfants. Ce chiffre ne prend pas en compte les 30 enfants prévus pour l'été 1962. Monsieur Étienne Dennery, Ambassadeur de la République Française en Suisse, à Monsieur le Ministre des Affaires Étrangères, Direction des Affaires politiques, Mission de Liaison avec l'Algérie, le 23 janvier 1962, AMAE, Secrétariat d'État aux affaires algériennes 1959-1967, carton 86. Aujourd'hui encore, il reste très difficile d'estimer le nombre d'enfants algériens transférés en Suisse pendant la guerre d'Algérie. Selon Jean-Claude Jaccard, Hamid Khatel, secrétaire de l'Association d'aide aux orphelins d'Algérie, aurait conclu un accord avec Renée Laborde, présidente des Euvres sociales de Philippeville, selon lequel 500 enfants algériens allaient être envoyés en Suisse par convois de 100. Cependant aucune trace de cet accord et de ses conséquences ne sont consultables à ce jour. Monsieur Jean-Claude Jaccard, Président de la «Terre des Hommes», au Ministre d’État chargé des Affaires algériennes, le 10 avril 1961, AMAE, Secrétariat d'État aux affaires algériennes 1959-1967, carton 86.

36 Le Ministre des Affaires étrangères à Monsieur le Premier Ministre (note 33),

37 Le Ministre d'État chargé des Affaires algériennes à Monsieur le Délégué général en Algérie, le 23 mars 1961, AMAE, Secrétariat d'État aux affaires algériennes 1959-1967, carton 86.

38 Le Ministre des Affaires étrangères à Monsieur le Premier Ministre (note 33). 
bien habillés au moment de leur arrivée ${ }^{(39)}$. Dans certains cas, le seul état de santé des mineurs aurait pu choquer leurs hôtes. Au mois de mai 1961, un tiers des quinze enfants envoyés en Suisse avait dû interrompre leur voyage pour être hospitalisés à Marseille avant de pouvoir continuer leur route ${ }^{(40)}$.

Malgré toutes ces difficultés, vers la fin de la guerre, les autorités françaises sur place se félicitaient de leur bonne coopération avec l'association de M. Jaccard tout en soulignant l'importance du rôle joué par les assistants sociaux. L'ambassadeur français retenait à ce sujet: "Les résultats obtenus en Suisse, grâce à leur action, et à celle de “Terre des Hommes”, ont été, jusqu’à présent, sur le plan humanitaire et sur le plan politique, extrêmement appréciables" ${ }^{(41)}$.

\section{Des jeunes de Bougie pour la paix en Europe, ou: Le mutisme ouest-allemand sur la guerre coloniale en Algérie}

Les séjours de jeunes Algériens en Allemagne de l'Ouest représentent un intérêt majeur en ce qu'ils illustrent, au niveau local, la position délicate de la République fédérale d'Allemagne par rapport à la guerre d'indépendance algérienne. Il faut préciser à ce stade que l'anticommunisme s'était à l'époque imposé comme l'un des piliers - si ce n'est le pilier central - de la politique du gouvernement de Konrad Adenauer. De ce fait, mais aussi dans l'optique de la construction d'une forte communauté européenne en partenariat avec Paris, le chancelier allemand fit pendant longtemps sienne cette fable française bien connue selon laquelle la rébellion en Algérie ne serait qu'un complot inspiré par Moscou ${ }^{(42)}$. Cependant, de plus en plus de membres du gouvernement remarquaient que ce serait justement cette guerre coloniale qui risquait d'entraîner une future Algérie indépendante dans le camp soviétique. Figé dans ce dilemme imposé par le contexte de la guerre froide, Bonn prenait la plupart du temps le parti de fermer les yeux sur les exactions de son allié français en Algérie ${ }^{(43)}$. Les quelques séjours d'adolescents algériens en RFA pendant la guerre d'Algérie montrent que l'occultation aussi bien du fait colonial que de la guerre en Algérie avait lieu également à un niveau local au profit d'un discours promouvant une Europe forte et unie.

L'organisation de ces voyages de jeunes Algériens en République fédérale d'Allemagne suivait la logique ainsi que la structure des jumelages européens évoqués plus haut. Le rôle central revenait à la ville portuaire algérienne Bejaïa, nommé Bougie durant l'époque coloniale. Par le biais du Conseil des communes en Europe, le conseil municipal de la commune de Bougie décidait le 11 octobre 1954 de se jumeler à la commune de Tübingen en RFA $^{(44)}$. Ce partenariat, auquel se joignirent peu après les

39 Le Ministre d'état chargé des Affaires algériennes à Monsieur le Délégué général en Algérie (note 37).

40 Télégramme du Préfet des Bouches-du-Rhône au Préfet de Constantine, le 6 mai 1961, AMAE, Secrétariat d'État aux affaires algériennes 1959-1967, carton 86.

41 Monsieur Étienne Dennery, Ambassadeur de la République Française en Suisse, à Monsieur le Ministre des Affaires étrangères (note 35).

42 CAHn/Müller, La République fédérale d'Allemagne et la guerre d'Algérie (note 5), p. 135-139.

43 Ibid.

44 Le Gouverneur général de l'Algérie à Monsieur le Ministre de l’Intérieur, Service de l'Algérie et des Départements d'Outre-Mer, Sous-Direction de l'Algérie, le 21 janvier 1955, AMAE, Secrétariat d'État aux affaires algériennes 1959-1967, carton 76. 
villes d'Ivrea en Italie, de Monthey en Suisse et de Jambes-Namur en Belgique ${ }^{(45)}$, représentait le seul cas de jumelage liant une ville allemande à une ville algérienne. À travers des voyages de jeunes dans la commune jumelée, c'est la solidité de ce partenariat qui devait se manifester. Au cours du mois d'août 1954, un groupe d'étudiants de Tübingen avait ainsi visité Bougie avant même le début officiel du jumelage. Un an après, un groupe de 26 adolescents de Bougie accompagnés de cinq moniteurs allaient à leur tour faire un séjour dans cette ville allemande de longue tradition universitaire. À cette occasion, le maire de Bougie, Jacques Augarde, se félicita d'avoir envoyé «une délégation de jeunes, jeunes d'origine européenne et chrétienne et jeunes de souche algérienne et musulmane, comme ambassadeurs [...] à travers l'Europe», considérant ce voyage comme une contribution à «la création de l'Eurafrique» ${ }^{(46)}$.

Mélangés à une délégation de 22 enfants originaires de Monthey, les jeunes de Bougie furent d'abord accueillis par le maire de Tübingen et un représentant des forces militaires françaises stationnées près de la ville ${ }^{(47)}$. Pendant ces huit jours, les invités firent la connaissance de scouts allemands et visitèrent, entre autres, les usines Mercedes à Stuttgart, l'université de Tübingen, ainsi que le château des Hohenzollern à quelques kilomètres de la ville ${ }^{(48)}$.

Parallèlement à ce voyage salué par la presse locale comme un grand «signal de paix» et une leçon pour les générations précédentes, un groupe de scouts de Tübingen, parti au même moment en direction de Bougie, fut confronté à la réalité de la guerre en Algérie. C'est à travers le rapport de ces scouts que les habitants de Tübingen purent accéder à une image des plus réalistes de ce à quoi pouvait ressembler la Kabylie au mois d'août 1955: «Maintenant nous sommes dans le territoire des "hors la loi". [...] Nous avons vu pour la première fois des camps militaires français, des barbelés; des véhicules blindés sont stationnés près des fontaines des villages et il y a des soldats partout ${ }^{(49)}$. Le contraste avec le séjour des jeunes de Bougie à Tübingen ne pouvait faire aucun doute.

Pour des raisons jusqu'à présent inconnues, le jumelage entre Bougie et Tübingen fut rompu en 1956 au profit de la ville de Bad Homburg. En 1954, le maire de cette importante ville thermale allemande avait été élu dans le comité exécutif du $C C E^{(50)}$, amorçant ainsi son engagement particulier dans le mouvement communal européen. Au mois d'octobre 1956, la mairie de Bad Homburg organisait, en coopération avec Francfort-sur-le-Main, le troisième congrès du CCE auquel participaient plus de 500 représentants de diverses communes ${ }^{(51)}$. C'est précisément à cette occasion que

45 Tübinger Chronik, 29 décembre 1954, Tübinger Stadtarchiv.

46 Jacques Augarde, Préface, in: Les jeunes de Bougie construisent L'Eurafrique de l'amitié, Châlons-surMarne, 1956 (sans auteur).

47 Tübinger Chronik, 4 août 1955, Tübinger Stadtarchiv.

48 Les jeunes de Bougie construisent l'Eurafrique de l'amitié (note 46), p. 7-19.

49 Traduit par l'auteur. Voir le rapport du groupe de scouts «Steppenfüchse»: Der graue Reiter, 23, décembre 1955, p. 39, Stadtarchiv Tübingen ZGS 64 Bougie.

50 Johannes Latsch, «"Ein neues Europa, eine neue Welt”. 50 Jahre Bad Homburger Städtepartnerschaften: Vom Gemeindetag 1956 bis heute», in : Stadt Bad Homburg v. d. Höhe (éd.), Aus dem Stadtarchiv. Vorträge zur Bad Homburger Geschichte 2006/2007, Wertheim, 2008, p. 35-74.

51 Ibid. 
Bougie fut intégrée à un nouveau jumelage qui allait la lier non seulement à Bad Homburg mais aussi à Cabourg en France métropolitaine, à Spa en Belgique, à Bad Mondorf au Luxembourg, à Mayrhofen en Autriche, à Chur en Suisse ainsi qu'à Terracine en Italie. Cette alliance entre huit municipalités, dépassant largement le cadre habituel de deux ou au maximum trois partenaires d'un jumelage, était présentée comme une œuvre pionnière exercée dans un «esprit européen» exemplaire.

Tout se passait comme si l'identité européenne d'une ville de Kabylie ne faisait aucun doute. Les huit maires impliqués pouvaient alors faire le serment:

«Nous, maires librement élus par nos concitoyens, jurons à ce jour solennellement de maintenir les liens entre nos administrations communales, de soutenir l'échange entre nos habitants à tous les niveaux, d'encourager l'esprit fraternel européen par une compréhension réciproque toujours plus élevée, d’unir nos efforts afin de contribuer par tous les moyens accessibles au succès de cette œuvre nécessaire de la paix et de la prospérité, de l'unité européenne» ${ }^{(52)}$.

La réunion du CCE à Bad Homburg fut ainsi pour le maire de Bougie, Jacques Augarde, l'occasion de faire reconnaître d'une manière officielle et au-delà du cadre politique franco-algérien l'identité française de sa ville.

Fidèles à leur serment, les différents membres de ce nouveau jumelage, et la municipalité de Bad Homburg en particulier, ne se contentèrent pas, par la suite, de simples déclarations d'intentions. Dans le but de revendiquer le lien étroit entre les huit communes, il fut une fois de plus entrepris de réunir certains de leurs adolescents. Dans cette optique, Bad Homburg organisait au mois d'août 1958 un «rassemblement international de la jeunesse». À l'occasion de cet événement, 41 adolescents, dont cinq de Bougie, rejoignirent la ville thermale du Taunus. Afin de permettre la communication entre les adolescents présents, âgés de 14 à 18 ans, des interprètes de langue française, allemande et italienne étaient sur place ${ }^{(53)}$. Toujours dans le cadre de cet événement, la ville de Bougie ainsi que les jeunes d'Algérie étaient présentés comme adhérents d'un «jumelage de communes européennes» si bien que ni les organisateurs, ni les journalistes allemands ne semblaient s'apercevoir de ce paradoxe ${ }^{(54)}$ et ce encore moins du fait que l'on célébrait la paix européenne avec des ressortissants d'un pays déjà lourdement accusé au niveau international de mener une guerre atroce en Afrique du Nord. Interrogée sur ce constat, une participante allemande de l'époque se souvient que «tout le monde» présent à Bad Homburg n'était pas sans savoir qu'il se passait «quelque chose» en Algérie à ce moment-là. Malgré cela, bien conscients qu'en abordant de telles thématiques, on prenait le risque d'entraver la bonne entente parmi les jeunes, les organisateurs veillèrent à ce qu'un esprit d'unité et de consensus dominât les différentes manifestations ${ }^{(55)}$.

52 Traduit par l'auteur. Écrit sans date et sans auteur, Archives municipales de Bad Homburg (AMBH), Archives non classés: Partnerschaft, 50 Jahre 1956-2006.

53 Articles de presse sans date et sans auteurs relatifs au «Internationales Jugendtreffen » à Bad Homburg en août 1958, AMBH, Archives non classés: Frau Kuning.

54 La Frankfurter Neue Presse écrivait sans aucune annotation que Bad Homburg allait se jumeler à deux villes françaises. Frankfurter Neue Presse, le 4 octobre 1956. 
À cette occultation de la guerre s'ajoutait une autre différence majeure par rapport aux voyages de mineurs algériens effectués en Suisse. Les archives et les témoignages ne font mention d'aucune trace, à Bad Homburg, d'un encadrement ou même d'une quelconque "assistance» spéciale à l'image des CTAM en France. Apparemment les organisateurs du « rassemblement international de la jeunesse » n'avaient reçu que très peu d'instructions quant aux mœurs musulmanes. Un journal local rapporta à ce propos qu'un étudiant algérien de 18 ans avait refusé de goûter au fameux «Rippchen mit Kraut», un plat régional de la Hesse à base de viande de porc et de choucroute, pour des raisons religieuses ${ }^{(56)}$.

Tous les jeunes étaient logés dans des familles d'accueil allemandes de sorte que le contact fut totalement dépourvu de surveillance politique. Dans un cas précis, des hôtes se trouvèrent même confrontés directement à des effets de la guerre d'Algérie sur les jeunes gens. En apprenant qu'il allait être logé avec un adolescent français, un jeune de Bougie protesta vivement, déclarant qu'il aurait perdu plusieurs membres de sa famille durant la guerre alors en cours. Néanmoins, du fait d'un manque de possibilités d'hébergement, Djamal B. dut, pendant la durée de son séjour à Bad Homburg, partager un lit double avec un jeune originaire de Cabourg qu'il semblait pourtant percevoir comme son ennemi ${ }^{(57)}$.

Bien qu'omniprésents dans certains esprits, le contexte colonial et encore plus celui de la guerre d'Algérie restèrent volontairement occultés à Bad Homburg. C'est ainsi que cinq jeunes de Kabylie se retrouvèrent dans une ville thermale allemande aux côtés d'adolescents belges, italiens, suisses, luxembourgeois et français face à un maire allemand les appelant à contribuer au dépassement des déchirements politiques de l'Europe et à créer son unité pour ne pas être écrasé entre l'Amérique et l'URSS ${ }^{(58)}$.

Le cas de Bad Homburg illustre à quel point, parmi les différentes raisons motivant dans les années cinquante des communes à conclure un jumelage, le prestige que pouvaient apporter de telles relations jouait un rôle important. Ce fut particulièrement vrai pour la ville thermale de la Hesse qui, à l'occasion de la réunion évoquée de 1956, se préparait à recevoir non seulement les maires de nombreuses villes européennes mais aussi le ministre fédéral des Affaires étrangères.

En 1963, une collecte pour les nécessiteux de Bougie connut un succès inattendu parmi la population de Bad Homburg. Par la suite, le maire Armin Klein se chargea personnellement d'accompagner le transport de plus de 200 sacs de vêtements par bateau jusqu'à Bougie. Constamment suivi par la presse locale, il y fut accueilli notamment par le futur ambassadeur algérien en RFA, Hafid Keramane, comme un "grand ami de l'Algérie» ${ }^{(59)}$. Cela n'empêcha pas Klein de déclarer quelques jours plus tard devant la presse ainsi que devant le nouveau maire de Bougie Said Sfayah que, malgré le fait que la ville de Bougie soit située de l'autre côté de la Méditerranée, il s'agissait pourtant en vérité d'une ville européenne appartenant à une nation européenne ${ }^{(60)}$.

56 AMBH, Archives non classés: Frau Kuning (note 53).

57 Entretien de l'auteur avec Ingrid K., 22 janvier 2015.

$58 \mathrm{AMBH}$, Archives non classés: Frau Kuning (note 53).

59 Frankfurter Neue Presse, le 9 mai 1963, AMBH, A $04-77$.

$60 \mathrm{AMBH}$, Archives non classés: Frau Kuning (note 53). 


\section{Conclusion}

L'organisation de séjours de mineurs algériens dans des colonies de vacances en France métropolitaine durant la guerre d'Algérie avait pour objectif de resserrer les liens entre la France et l'Algérie au niveau local et ainsi de contrecarrer l'action du FLN. En conséquence, ils doivent être compris avant tout comme un réseau créé pour servir de propagande au maintien de l'Algérie française ${ }^{(61)}$. Les différentes activités réalisées dans le cadre du parrainage Moselle-Blida et ensuite du jumelage Metz-Blida illustrent non seulement l'intégration de multiples acteurs métropolitains locaux dans ce projet, mais aussi l'importance donnée au ralliement des enfants et adolescents français et algériens au projet colonial mis en difficultés.

Pour ce qui est des voyages de mineurs algériens dans des pays limitrophes de la France, il s'agit d'apporter une analyse plus nuancée. Les cas suisses et ouest-allemands étudiés montrent que les acteurs impliqués avaient leurs propres motifs pour accueillir des enfants algériens. En premier lieu, on relèvera le désir de venir en aide à des enfants vivant dans des conditions déplorables tant au niveau social que sanitaire. Ce but, ayant été atteint au moins dans certains cas, n'empêche pas de constater que l'armée française appréciait l'intervention de certaines organisations privées helvétiques. D’ordinaire, l'action humanitaire non gouvernementale ne peut se passer d'une certaine collaboration avec des acteurs étatiques luttant pour la souveraineté d'un territoire, ce qui ne permet pourtant pas de conclure à une instrumentalisation unilatérale.

Un autre motif ayant pu faciliter l'accueil de jeunes Algériens dans un pays voisin de la France durant la guerre d'Algérie nous apparaît à travers l'exemple du jumelage entre Bad Homburg et Bougie ou Bejaïa. Dans le cas de cette ville thermale allemande, le prestige attribué à une large connexion internationale de la municipalité semble avoir été le facteur décisif menant à la conclusion d'une liaison avec une ville algérienne. Les principaux acteurs ouest-allemands de ce jumelage germano-algérien ne concentraient pas leurs éloges sur l'Algérie française, mais plutôt, dans le contexte de la guerre froide, sur la revendication d'une Europe forte et unie face à l'Union soviétique. Ainsi, ni la présence de jeunes Algériens à Bad Homburg, dont certains étaient fortement marqués par le conflit colonial, ni la conclusion de l'indépendance algérienne n'ont conduit la municipalité à aborder cette problématique aux dépens de son discours pro-européen.

Dans une perspective plus générale, l’organisation de séjours pour des jeunes Algériens en Europe montre une fois de plus la nécessité d'étudier la guerre d'indépendance algérienne à la lumière de sa dimension internationale. Il est bien connu que ni les partisans de l'Algérie française, ni ceux de l'indépendance algérienne ne limitaient leurs actions au territoire algérien et français. Il convient cependant de rappeler que les acteurs impliqués s'appropriaient les différents clivages de ce conflit à leur propre façon et selon leurs propres intérêts, interprétations et stratégies. Une telle perspective devrait permettre de sortir davantage des raisonnements binaires dans lesquels ce conflit est encore trop souvent présenté.

61 Gérard Bourdin, «Les ambiguïtés d'un rapprochement: parrainages et jumelages, 1956-1962», in: Raphaëlle Branche, Sylvie Thénault (éd.), La France en guerre, 1954-1962. Expériences métropolitaines de la guerre d'indépendance algérienne, Paris, Autrement (coll. Mémoires/Histoire), 2008, p. 82-100; L. HARDT, «Ferienlager gegen die Rebellion» (note 2). 


\section{Résumé}

Cet article se penche sur les voyages d'enfants et d'adolescents algériens en Suisse, en RFA et en France métropolitaine durant la guerre d'indépendance algérienne (19541962). Dans ces trois pays, différents acteurs se sont efforcés de permettre à des garçons et des filles algériens de quitter la zone de combat pour venir en Europe, le temps d'un séjour en colonie de vacances ou dans des familles d'accueil. Malgré l'obligation pour les organisateurs de coopérer avec les autorités françaises, leurs motivations étaient bien différentes. Tandis qu'une action psychologique pour le maintien de l'Algérie française dominait dans les colonies de vacances en métropole, une propagande dirigée contre l'Union soviétique et favorisant une identité européenne prévalait durant les séjours en Allemagne de l'Ouest. En Suisse, cependant, c'était l'aspect humanitaire qui jouait un rôle central. Ces différents objectifs marquaient aussi le déroulement des séjours des mineurs algériens concernés comme le montrent les échanges réalisés dans le cadre des jumelages entre Metz et Blida, Bad Homburg et Bougie ainsi que les activités des organisations helvétiques «Pour Aïn-Touta» et «Terre des Hommes».

\section{Zusammenfassung}

Dieser Artikel befasst sich mit den Reisen algerischer Kinder und Jugendlicher in die Schweiz, in die BRD und nach Frankreich während des algerischen Unabhängigkeitskrieges (1954-1962). In den drei Ländern bemühten sich verschiedene Akteure darum, algerische Jungen und Mädchen für eine begrenzte Zeit aus dem Kriegsgebiet nach Westeuropa zu holen, um sie etwa in Gastfamilien oder in Ferienlagern unterzubringen. Obwohl die Organisatoren dabei stets auch mit den französischen Behörden kooperieren mussten, waren ihre jeweiligen Motivationslagen für diese Aktionen sehr unterschiedlich gelagert. Während in Frankreich vor allem auf den Erhalt der „Algérie française“ hingearbeitet wurde, stand in der BRD die Festigung eines starken Europas in Abgrenzung zur Sowjetunion im Vordergrund. In der Schweiz spielten vor allem humanitäre Aspekte eine zentrale Rolle. Dass sich diese Ziele auch in der konkreten Gestaltung der jeweiligen Aufenthalte algerischer Kinder und Jugendlicher spiegelten, wird am Beispiel der Austauche gezeigt, die im Rahmen der Partnerschaften von Metz und Blida, Bad Homburg und Bougie stattfanden, sowie den Aktivitäten der Organisationen „Pour Aïn-Touta" und „Terre des Hommes".

\footnotetext{
Abstract

During the Algerian war of independence (1954-1962) Algerian children and teenagers were sent to France, Western-Germany and Switzerland. In these three countries different actors managed to get Algerian boys and girls out of the war zone and provided them short stays in European holiday camps or host families. Even though all of the organizers had to cooperate with the French authorities, their motivations differed widely. While a psychological action for recovering the "Algérie française" prevailed in metropolitan France, a pro-European and anti-communist propaganda predominated in western Germany. In Switzerland humanitarian action was the most important motive for receiving Algerian minors. These disparate incitements had an important impact on the course of the Algerians short-stays in western Europe as it is shown referring to activities of the Swiss NGO's "Pour Ain-Touta" and "Terre des Hommes" and the exchanges between the twin towns Metz and Blida, Bad Homburg and Bougie.
} 